\title{
An analytical solution to the Graetz problem with viscous dissipation for non-Newtonian fluids
}

\author{
R. Chiba ${ }^{1}$, M. Izumi ${ }^{2} \&$ Y. Sugano ${ }^{3}$ \\ ${ }^{1}$ Miyagi National College of Technology, Japan \\ ${ }^{2}$ Department of Mechanical Engineering, \\ Ishinomaki Sensyu University, Japan \\ ${ }^{3}$ Department of Mechanical Engineering, Iwate University, Japan
}

\begin{abstract}
Forced convection heat transfer in a non-Newtonian fluid flow between parallel plates subjected to convective cooling on the external surfaces is investigated analytically. Fully developed laminar velocity distributions obtained by a power law fluid rheology model are used, and viscous dissipation is taken into account. The effect of heat conduction in the direction of fluid flow is considered negligible. The physical properties are assumed to be constant. We approximate the smooth change in the velocity distribution between the plates as a piecewise constant velocity. The theoretical analysis of the heat transfer is performed using an integral transform technique-Vodicka's method. An important feature of the approach is that an arbitrary distribution of the temperatures of the surrounding media in the direction of fluid flow and an arbitrary velocity distribution of the fluid can be permitted. A comparison with the existing results provides a verification of this technique. The effects of the Brinkman number, Biot number and rheological properties on the distributions of the fluid temperature and the local Nusselt number are illustrated. Moreover, the effects of these parameters on the length of the freeze-free zone are discussed in the case where the temperatures of the surrounding media are below the solidification temperature of the fluid.

Keywords: heat transfer, forced convection, non-Newtonian fluid, analytical solution, viscous dissipation, Graetz problem, channel flow.
\end{abstract}




\section{Introduction}

An understanding of the convection heat transfer in non-Newtonian fluids inside conduits is of great importance in the design of several types of thermal equipment. From this viewpoint, heat transfer problems of this type have been investigated by a large number of researchers since the 1880s. The problem pertaining to the derivation of the local Nusselt number in the thermal entry region when an incompressible fluid flows through a conduit with a fully developed velocity distribution is of particular interest; this problem is referred to as the Graetz problem. It has attracted the interest of not only engineers but also applied mathematicians because of the difficulties involved in deriving the solution.

The Graetz problem is normally solved by using a quasi-analytical method. That is, the separation of variables approach is applied to the governing equation, and only the resultant eigenvalue problem is solved numerically. It is very difficult to obtain an exact solution to the eigenvalue problem except in some special cases. Therefore, a completely explicit analytical solution to the heat transfer problem for a fluid with an arbitrary velocity distribution has not been reported thus far.

The objective of this study is to solve mathematically the forced convection heat transfer problem in a conduit between parallel plates subjected to heat loads from the surrounding by Vodicka's method [1], which is a type of integral transform method, and to derive completely explicit analytical solutions of the fluid temperature and local Nusselt number. Heat conduction in the direction of fluid flow is considered negligible since the present study focuses on heat transfer with a sufficiently large Peclet number. However, viscous dissipation is taken into account. Numerical calculations are used to illustrate the effects of the Brinkman number, Biot number and rheological properties on the distributions of the fluid temperature and local Nusselt number. Moreover, the effects of these parameters on the length of the freeze-free zone are discussed for the case where the temperatures of the surrounding media are below the solidification temperature of the fluid.

\section{Analysis}

\subsection{Analytical model and formulation}

Figure 1 shows the physical model and coordinate system. A non-Newtonian fluid with a fully developed velocity distribution $u(y)$ flows into a conduit between parallel plates. The fluid temperature at the entrance is $T_{0}(y)$. The conduit of width $2 L$ contacts the surrounding media with temperature $T_{1 \infty}(x)$ and $T_{2 \infty}(x)$ at $y=L$ and $y=-L$, respectively. The heat transfer coefficients on the external surfaces of the plates are $h_{1}$ and $h_{2}$.

In this study, the flowing fluid has no analytical restriction in its velocity distribution form. With regard to the type of fluid flowing inside the conduit, a power law fluid, which can approximate the non-Newtonian viscosity of many 
types of fluids with a good accuracy over a wide range of shear rates, is considered here. The shear stress acting on the viscous fluid $\tau_{y x}$ is given as follows:

$$
\tau_{y x}=\kappa\left|\frac{\mathrm{d} u}{\mathrm{~d} y}\right|^{\nu-1} \frac{\mathrm{d} u}{\mathrm{~d} y},
$$

where $\kappa$ and $v$ are the power law model parameter and power law model index, respectively. $v<1$ indicates a pseudoplastic fluid, $v>1$ indicates a dilatant fluid and $v=1$ is equivalent to a Newtonian fluid. The fully developed velocity distribution is expressed as follows [2]:

$$
u(y)=u_{m} \frac{2 v+1}{v+1}\left(1-\left|\frac{y}{L}\right|^{\frac{1+v}{v}}\right),
$$

where $u_{\mathrm{m}}$ is the mean velocity.

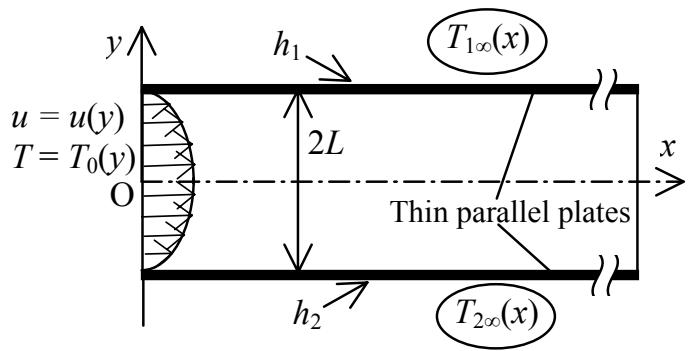

Figure 1: Physical model and coordinate system.

The following assumptions are introduced:

(i) material properties are independent of temperature and are therefore constant,

(ii) heat resistance of the parallel plates is negligible,

(iii) heat conduction in the direction of fluid flow is negligible,

(iv) mode of flow is always laminar.

In this case, the steady-state heat balance taking viscous dissipation into account is expressed as follows:

$$
\rho c u \frac{\partial T}{\partial x}=\lambda \frac{\partial^{2} T}{\partial y^{2}}+\tau_{y x} \frac{\mathrm{d} u}{\mathrm{~d} y},
$$

where $\rho, c$ and $\lambda$ are the density, specific heat and thermal conductivity, respectively. The boundary conditions are given as follows:

$$
\begin{gathered}
T(0, y)=T_{0}(y), \\
\lambda \frac{\partial T(x, L)}{\partial y}+h_{1}\left[T(x, L)-T_{1 \infty}(x)\right]=0,
\end{gathered}
$$


26 Advanced Computational Methods in Heat Transfer IX

$$
\lambda \frac{\partial T(x,-L)}{\partial y}-h_{2}\left[T(x,-L)-T_{2 \infty}(x)\right]=0 .
$$

Taking the generality of the analysis into account, the expressions of eqns (3)-(6) in dimensionless form yield the following equations:

$$
\begin{gathered}
U(\eta) \frac{\partial \theta}{\partial \xi}=\frac{\partial^{2} \theta}{\partial \eta^{2}}+B r\left(\frac{1+v}{v}\right)^{v+1}|\eta|^{\frac{v+1}{v}}, \\
\theta(0, \eta)=\theta_{0}(\eta), \\
\frac{\partial \theta(\xi, 1)}{\partial \eta}+H_{1}\left[\theta(\xi, 1)-\theta_{1 \infty}(\xi)\right]=0, \\
\frac{\partial \theta(\xi,-1)}{\partial \eta}-H_{2}\left[\theta(\xi,-1)-\theta_{2 \infty}(\xi)\right]=0,
\end{gathered}
$$

where $U(\eta)=(2 v+1) /(v+1)\left[1-|\eta|^{(1+v) / v}\right], B r=\kappa u_{\max }^{1+v} L^{1-v} /\left[\lambda\left(T_{0 \mathrm{~b}}-T_{\mathrm{s}}\right)\right]$, $u_{\max }=(1+2 v) u_{\mathrm{m}} /(1+v), \theta=\left(T-T_{\mathrm{s}}\right) /\left(T_{0 \mathrm{~b}}-T_{\mathrm{s}}\right), \eta=y / L, \xi=\lambda x /\left(u_{\mathrm{m}} L^{2} \rho c\right)$ and $H=$ $h L / \lambda$. Eqn (7) is a partial differential equation with variable coefficients; therefore, it is very difficult to obtain the exact solution. In order to solve eqn (7), we divide the conduit into $n$ regions in the $\eta$ direction and approximate $U(\eta)$ as a constant $U_{i}$ in each region, as shown in fig. 2 . In this case, the dimensionless energy equation in the $i$ th region $(i=1,2, \ldots, n)$ is obtained as follows:

$$
U_{i} \frac{\partial \theta_{i}}{\partial \xi}=\frac{\partial^{2} \theta_{i}}{\partial \eta^{2}}+Q(\eta),
$$

where $Q(\eta)=\operatorname{Br}[(1+v) / v]^{v+1}|\eta|^{(1+v) / v}$. The continuous conditions at the imaginary interfaces and boundary conditions are expressed by the following equations:

$$
\begin{gathered}
\theta_{i}\left(\xi, \eta_{i}\right)=\theta_{i+1}\left(\xi, \eta_{i}\right), \\
\frac{\partial \theta_{i}\left(\xi, \eta_{i}\right)}{\partial \eta}=\frac{\partial \theta_{i+1}\left(\xi, \eta_{i}\right)}{\partial \eta}, \\
\theta_{i}(0, \eta)=\theta_{0}(\eta),
\end{gathered}
$$

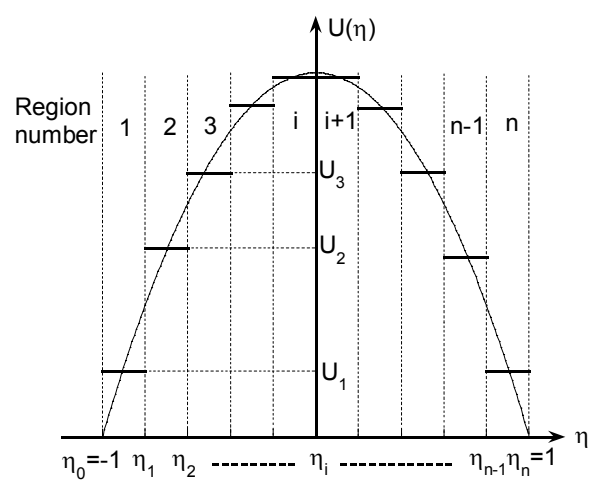

Figure 2: Virtual division inside the conduit. 


$$
\begin{gathered}
\frac{\partial \theta_{n}(\xi, 1)}{\partial \eta}+H_{1}\left[\theta_{n}(\xi, 1)-\theta_{1 \infty}(\xi)\right]=0, \\
\frac{\partial \theta_{1}(\xi,-1)}{\partial \eta}-H_{2}\left[\theta_{1}(\xi,-1)-\theta_{2 \infty}(\xi)\right]=0 .
\end{gathered}
$$

\subsection{Vodicka's method}

The solution to eqns (11)-(16) is obtained as

$$
\theta_{i}(\xi, \eta)=\sum_{m=1}^{\infty} \phi_{m}(\xi) X_{i m}(\eta)+\sum_{j=1}^{2} L_{j}(\eta) P_{j}(\xi)
$$

where

$$
P_{1}(\xi)=-\theta_{2 \infty}(\xi), P_{2}(\xi)=\theta_{1 \infty}(\xi) .
$$

$L_{j}(\eta)(j=1,2)$ is the solution to the boundary value problem expressed as follows:

$$
\frac{\mathrm{d}^{2} L_{j}}{\mathrm{~d} \eta^{2}}=0, \frac{\mathrm{d} L_{j}(-1)}{\mathrm{d} \eta}-H_{2} L_{j}(-1)=H_{2} \cdot(2-j), \frac{\mathrm{d} L_{j}(1)}{\mathrm{d} \eta}+H_{1} L_{j}(1)=H_{1} \cdot(j-1) .
$$

$X_{i m}(\eta)$ is the solution to the eigenvalue problem expressed as follows:

$$
\begin{gathered}
\frac{\mathrm{d}^{2} X_{i m}}{\mathrm{~d} \eta^{2}}+\gamma_{m}^{2} U_{i} X_{i m}=0, X_{i m}\left(\eta_{i}\right)=X_{(i+1) m}\left(\eta_{i}\right), \frac{\mathrm{d} X_{i m}\left(\eta_{i}\right)}{\mathrm{d} \eta}=\frac{\mathrm{d} X_{(i+1) m}\left(\eta_{i}\right)}{\mathrm{d} \eta}, \\
\frac{\mathrm{d} X_{1 m}(-1)}{\mathrm{d} \eta}-H_{2} X_{1 m}(-1)=0, \frac{\mathrm{d} X_{n m}(1)}{\mathrm{d} \eta}+H_{1} X_{n m}(1)=0 .
\end{gathered}
$$

$L_{j}(\eta)$ and $X_{i m}(\eta)$ are given as

$$
\begin{gathered}
L_{1}(\eta)=\frac{H_{1} H_{2} \eta-\left(1+H_{1}\right) H_{2}}{H_{1}+H_{2}+2 H_{1} H_{2}}, L_{2}(\eta)=\frac{H_{1} H_{2} \eta+\left(1+H_{2}\right) H_{1}}{H_{1}+H_{2}+2 H_{1} H_{2}}, \\
X_{i m}(\eta)=A_{i m} \cos \left(\sqrt{U_{i}} \gamma_{m} \eta\right)+B_{i m} \sin \left(\sqrt{U_{i}} \gamma_{m} \eta\right) .
\end{gathered}
$$

The conditions necessary to determine the unknown coefficients $A_{i m}$ and $B_{i m}$ can be obtained by substituting eqn (22) into the continuous and boundary conditions in eqn (20). Eigenvalues $\gamma_{m}(m=1,2, \ldots)$ are obtained from the condition under which $A_{i m}$ and $B_{i m}$ are both non-zero and are therefore positive roots of the following transcendental equation:

$$
\mathbf{G}_{\mathrm{e} 1} \cdot \mathbf{E}_{\mathrm{e} 1} \cdot \mathbf{E}_{\mathrm{e} 2} \cdots \mathbf{E}_{\mathrm{e}(n-1)} \cdot \mathbf{a}_{\mathrm{e} n}=0,
$$

where

$$
\begin{aligned}
& \mathbf{G}_{\mathrm{e} 1}=\left[d_{1 m} \sin \left(d_{1 m}\right)-H_{2} \cos \left(d_{1 m}\right) \quad d_{1 m} \cos \left(d_{1 m}\right)+H_{2} \sin \left(d_{1 m}\right)\right], d_{i m}=\sqrt{U_{i}} \gamma_{m}, \\
& \mathbf{E}_{\mathrm{e} i}=\left[\begin{array}{cc}
d_{i m} \cos \left(d_{i m} \eta_{i}\right) & -\sin \left(d_{i m} \eta_{i}\right) \\
d_{i m} \sin \left(d_{i m} \eta_{i}\right) & \cos \left(d_{i m} \eta_{i}\right)
\end{array}\right]\left[\begin{array}{cc}
\cos \left[d_{(i+1) m} \eta_{i}\right] & \sin \left[d_{(i+1) m} \eta_{i}\right] \\
-d_{(i+1) m} \sin \left[d_{(i+1) m} \eta_{i}\right] & d_{(i+1) m} \cos \left[d_{(i+1) m} \eta_{i}\right]
\end{array}\right],
\end{aligned}
$$


28 Advanced Computational Methods in Heat Transfer IX

$$
\mathbf{a}_{\mathrm{e} n}=\left[\begin{array}{l}
d_{n m} \cos \left(d_{n m}\right)+H_{1} \sin \left(d_{n m}\right) \\
d_{n m} \sin \left(d_{n m}\right)-H_{1} \cos \left(d_{n m}\right)
\end{array}\right] .
$$

By substituting eqn (17) into eqn (14), the following equation is obtained:

$$
G(\eta)=\sum_{m=1}^{\infty} \phi_{m}(0) X_{i m}(\eta)=\theta_{0}(\eta)-\sum_{j=1}^{2} L_{j}(\eta) P_{j}(0) .
$$

The eigenvalue function $X_{i m}(\eta)$ obtained from eqns (20) and (22) has an orthogonal relationship with discontinuous weight functions; this is expressed as follows:

$$
\sum_{i=1}^{n} U_{i} \int_{\eta_{i-1}}^{\eta_{i}} X_{i m}(\eta) X_{i k}(\eta) \mathrm{d} \eta=\left\{\begin{array}{cc}
\text { const. } & (m=k) \\
0 & (m \neq k)
\end{array} .\right.
$$

$L_{j}(\eta), Q(\eta)$ and $G(\eta)$ can be expanded into an infinite series by $X_{i m}(\eta)$ as follows:

$$
\begin{aligned}
& L_{j}(\eta)=\sum_{m=1}^{\infty} l_{m j} X_{i m}(\eta), \\
& Q(\eta)=U_{i} \sum_{m=1}^{\infty} q_{m} X_{i m}(\eta), \\
& G(\eta)=\sum_{m=1}^{\infty} g_{m} X_{i m}(\eta),
\end{aligned}
$$

where

$$
\begin{gathered}
l_{m j}=M_{m}^{-1} \sum_{i=1}^{n} U_{i} \int_{\eta_{i-1}}^{\eta_{i}} L_{j}(\eta) X_{i m}(\eta) \mathrm{d} \eta, q_{m}=M_{m}^{-1} \sum_{i=1}^{n} \int_{\eta_{i-1}}^{\eta_{i}} Q(\eta) X_{i m}(\eta) \mathrm{d} \eta, \\
g_{m}=M_{m}^{-1} \sum_{i=1}^{n} U_{i} \int_{\eta_{i-1}}^{\eta_{i}} G(\eta) X_{i m}(\eta) \mathrm{d} \eta, M_{m}=\sum_{i=1}^{n} U_{i} \int_{\eta_{i-1}}^{\eta_{i}}\left[X_{i m}(\eta)\right]^{2} \mathrm{~d} \eta .
\end{gathered}
$$

Taking eqns (19) and (20) into account, we substitute eqns (17), (27) and (28) into eqn (11). This yields a first-order linear ordinary differential equation for $\phi_{m}(\xi)$ as follows:

$$
\frac{\mathrm{d} \phi_{m}}{\mathrm{~d} \xi}+\gamma_{m}^{2} \phi_{m}=q_{m}-\sum_{j=1}^{2} l_{m j} \frac{\mathrm{d} P_{j}}{\mathrm{~d} \xi} .
$$

Solving eqn (31) with the condition $\phi_{m}(0)=g_{m}$, which is obtained from the comparison between eqns (25) and (29), we obtain $\phi_{m}(\xi)$ as

$$
\phi_{m}(\xi)=g_{m} e^{-\gamma_{m}^{2} \xi}+\frac{q_{m}}{\gamma_{m}^{2}}\left(1-e^{-\gamma_{m}^{2} \xi}\right)-e^{-\gamma_{m}^{2} \xi} \int_{0}^{\xi} e^{\gamma_{m}^{2} t} \sum_{j=1}^{2} l_{m j} \frac{\mathrm{d} P_{j}(t)}{\mathrm{d} t} \mathrm{~d} t .
$$

Finally, the temperature solution for the $i$ th region inside the conduit $\theta_{i}(\xi, \eta)$ is derived as follows:

$$
\begin{aligned}
& \theta_{i}(\xi, \eta)=\sum_{m=1}^{\infty} \phi_{m}(\xi)\left[A_{i m} \cos \left(\sqrt{U_{i}} \gamma_{m} \eta\right)+B_{i m} \sin \left(\sqrt{U_{i}} \gamma_{m} \eta\right)\right] \\
& +\frac{\left[\left(1+H_{1}\right) H_{2}-H_{1} H_{2} \eta\right] \theta_{2 \infty}(\xi)+\left[\left(1+H_{2}\right) H_{1}+H_{1} H_{2} \eta\right] \theta_{1 \infty}(\xi)}{H_{1}+H_{2}+2 H_{1} H_{2}}
\end{aligned} .
$$


The dimensionless bulk temperature of the fluid is given as

$$
\theta_{\mathrm{B}}(\xi)=\frac{1}{2} \int_{-1}^{1} U(\eta) \theta(\xi, \eta) \mathrm{d} \eta
$$

Consequently, the local Nusselt numbers at $\eta=1$ and -1 are expressed by the following equations:

$$
\begin{aligned}
& N u_{1}(\xi)=\frac{\hat{h}_{1} L}{\lambda}=\frac{\frac{\partial \theta_{n}(\xi, 1)}{\partial \eta}}{\theta_{n}(\xi, 1)-\theta_{\mathrm{B}}(\xi)}=\frac{-1}{\theta_{n}(\xi, 1)-\theta_{\mathrm{B}}(\xi)} \\
& \times\left\{\sum_{m=1}^{\infty} \phi_{m}(\xi) \sqrt{U_{n}} \gamma_{m}\left[A_{n m} \sin \left(\sqrt{U_{n}} \gamma_{m}\right)-B_{n m} \cos \left(\sqrt{U_{n}} \gamma_{m}\right)\right]-\frac{H_{1} H_{2}\left[\theta_{1 \infty}(\xi)-\theta_{2 \infty}(\xi)\right]}{H_{1}+H_{2}+2 H_{1} H_{2}}\right\}, \\
& N u_{2}(\xi)=\frac{\hat{h}_{2} L}{\lambda}=\frac{-\frac{\partial \theta_{1}(\xi,-1)}{\partial \eta}}{\theta_{1}(\xi,-1)-\theta_{\mathrm{B}}(\xi)}=\frac{-1}{\theta_{1}(\xi,-1)-\theta_{\mathrm{B}}(\xi)} \\
& \times\left\{\sum_{m=1}^{\infty} \phi_{m}(\xi) \sqrt{U_{1}} \gamma_{m}\left[A_{1 m} \sin \left(\sqrt{U_{1}} \gamma_{m}\right)+B_{1 m} \cos \left(\sqrt{U_{1}} \gamma_{m}\right)\right]+\frac{H_{1} H_{2}\left[\theta_{1 \infty}(\xi)-\theta_{2 \infty}(\xi)\right]}{H_{1}+H_{2}+2 H_{1} H_{2}}\right\} .
\end{aligned}
$$

\section{Numerical calculation}

As a numerical example, we consider the case of $\theta_{0}(\eta)=1, \theta_{1 \infty}(\xi)=\theta_{2 \infty}(\xi)=0$ and $H_{1}=H_{2}=H$. In this case, the local Nusselt numbers of eqns (35) and (36) equate with each other since the temperature field in the conduit is symmetric with respect to the $x$-axis ( $\xi$-axis).

The number of terms in the infinite series in eqn (33) is 500, unless otherwise specified. Note that this value is used under the verification of a sufficient convergence of the numerical results.

\section{Results and discussion}

\subsection{Examination of the number of partitions}

In order to estimate the accuracy and usefulness of the present analytical solution, which is obtained from the approximation of continuous change as a piecewise constant in the fluid velocity distribution, we first consider the most basic Graetz problem of $B r=0, H=\infty$ and $v=1$, or the case in which a Newtonian fluid flows without viscous dissipation between parallel plates that are maintained at a constant temperature. This case has already been analyzed quasi-analytically by Rosales et al. [3].

Figure 3 shows a comparison of the fluid temperature distribution calculated by the present solution with that in reference [3]. A smaller value of $\xi$ has a larger effect of the number of partitions $n$ on the temperature distribution. For $n=5$, the temperature distribution at $\xi=10^{-4}$ fluctuates widely. The temperature distribution obtained from the present analytical solution with $n=20$ 
is in good agreement with that of reference [3] for all $\xi$. Table 1 shows a comparison between the local Nusselt numbers of the present solution and reference [3]. For $n=20$, the difference between them is less than $0.1 \%$ in the range of $10^{-4}<\xi<10^{0}$. Consequently, as long as the local Nusselt number is discussed in this range, $n=20$ provides sufficiently accurate results. Note that all of the following results are obtained from the analytical solution with $n=20$.

Table 1: Comparison of local Nusselt numbers.

\begin{tabular}{|c|c|c|c|}
\hline \multirow{2}{*}{$\xi$} & \multirow{2}{*}{$\begin{array}{c}\text { Number of } \\
\text { eigenvalues used }\end{array}$} & \multicolumn{2}{|c|}{$\mathrm{Nu}$} \\
\cline { 3 - 4 } & 500 & {$[$ Rosales et al. $]$} & {$[$ Present $(n=20)]$} \\
\hline $10^{-4}$ & 200 & 7.7513 & 16.6615 \\
\hline $10^{-3}$ & 200 & 3.6934 & 7.7492 \\
\hline $10^{-2}$ & 200 & 2.04782 & 3.6926 \\
\hline $10^{-1}$ & 200 & 1.88517 & 2.04766 \\
\hline $10^{0}$ & \multicolumn{3}{|c}{} \\
\hline
\end{tabular}

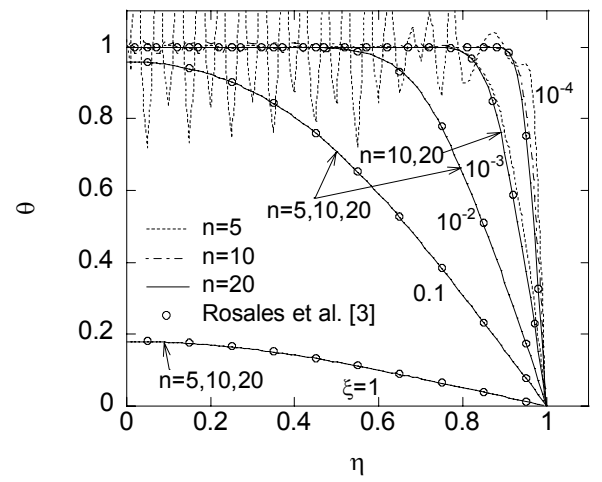

Figure 3: Relationship between the number of partitions $n$ and convergence of dimensionless temperature $\theta$.

\subsection{Effects of parameters on local Nusselt number distribution}

Figure 4 illustrates the effects of the viscous dissipation, heat transfer coefficients of the external surfaces and rheological character of the fluid on the local Nusselt number around the entrance of the conduit. The Nusselt numbers at $\xi=10^{-4}$ are the largest in figs. 4(a) and (b). For the Brinkman number $\mathrm{Br}=0$, the Nusselt numbers decrease simply with an increase in $\xi$ and finally converge on the Nusselt numbers in the developed temperature field. On the other hand, for $B r \neq 0$, the Nusselt numbers do not necessarily decrease monotonously, and they show a change from a decreasing to an increasing trend, especially for small values of $\mathrm{Br}$. However, they converge to a certain value, regardless of the value of $B r$. While the Nusselt numbers at $\xi=10^{-4}$ increase with a decrease in the Biot number $H$, the convergent value of the Nusselt number for $B r \neq 0$ is 
independent of the Biot number, only depending on $v$. Smaller values of $v$ tend to result in higher local Nusselt numbers throughout the conduit.
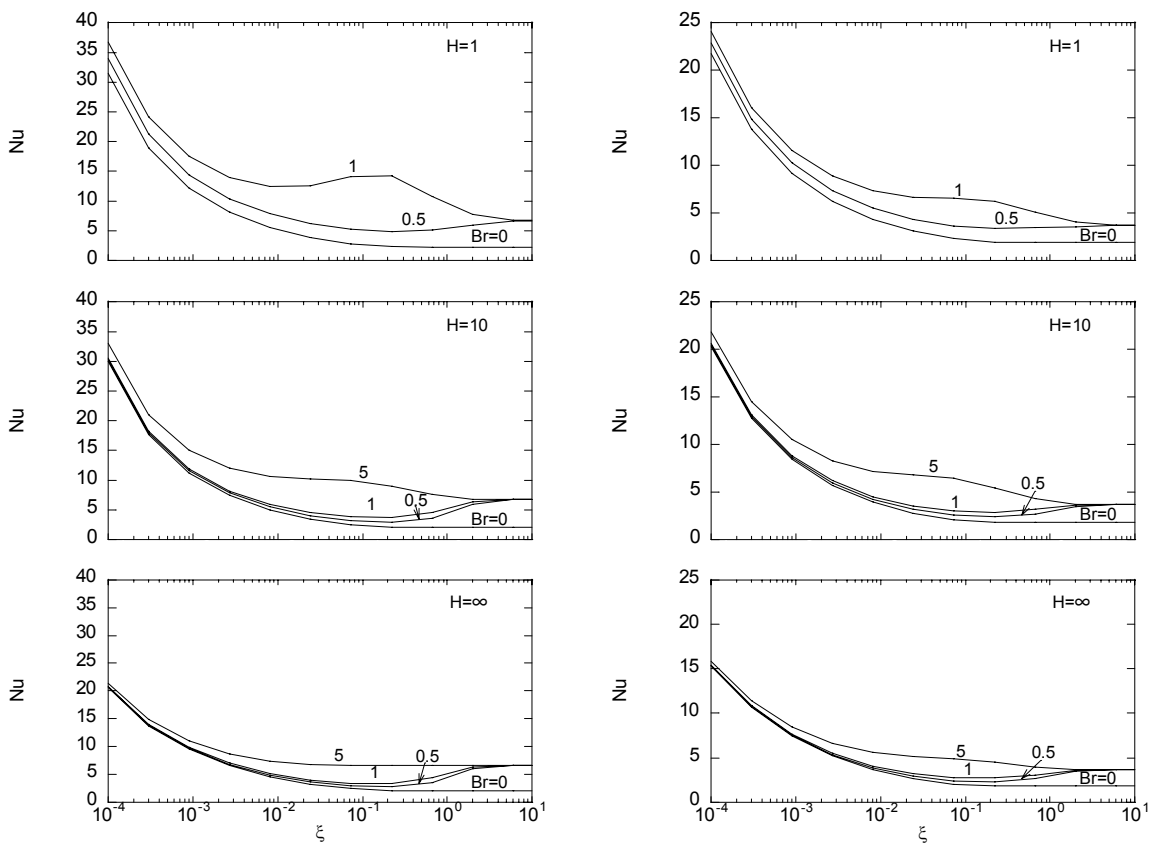

(a) $v=0.3$

(b) $v=3$

Figure 4: Nusselt number around the channel entrance.

\subsection{Critical fluid velocity diagram}

The critical fluid velocity for avoiding solidification within the conduit is obtained in the case where the temperatures of the surrounding media are below the solidification temperature of the flowing fluid; this highlights the applicability of the present analytical solution in the field of industry.

Figure 5 shows the relationship between the dimensionless solidification temperature and the dimensionless length of the freeze-free zone with different Biot numbers and Brinkman numbers. This figure makes use of the temperature distribution in the direction of the $\xi$ coordinate at $\eta=1$. For $B r=0$, it can be seen that $\theta_{\mathrm{f}}$ is asymptotic to 1 as $\xi_{\mathrm{f}}$ approaches 0 and $\theta_{\mathrm{f}}$ is asymptotic to 0 as $\xi_{\mathrm{f}}$ approaches infinity. The extent of the approach depends on the value of $H$. This result is the same as that reported by Sadeghipour et al. [4], who investigated the critical fluid velocity of a pipe flow. For $B r \neq 0$, the effect of $B r$ becomes significant as $H$ decreases and $\xi_{\mathrm{f}}$ increases. The effect of $v$ on the critical fluid velocity is considerably smaller than that of $\mathrm{H}$ or $\mathrm{Br}$. 

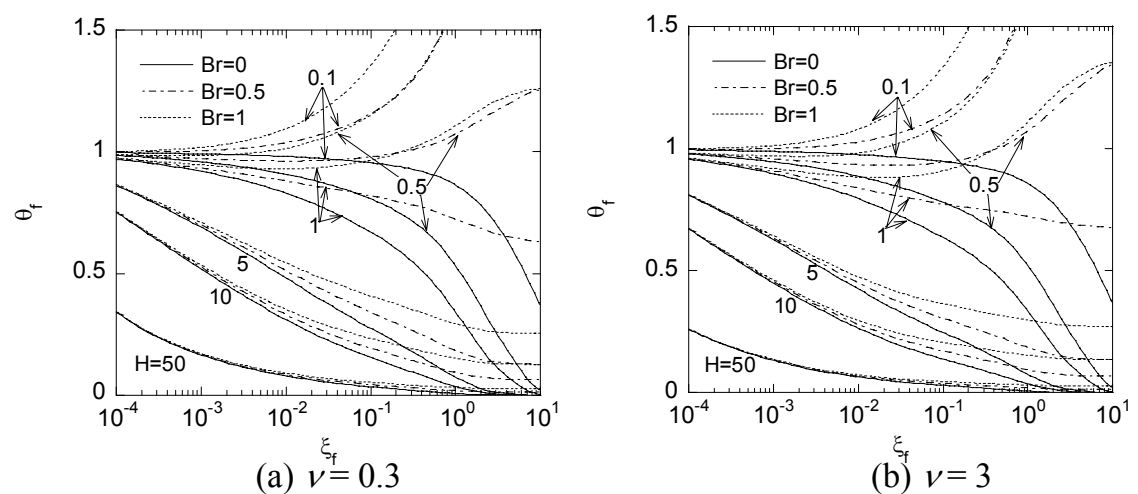

Figure 5: Variation of freeze-free zone with dimensionless solidification temperature for different Biot numbers and Brinkman numbers.

\section{Conclusions}

The forced convection heat transfer problem with viscous dissipation in a conduit between parallel plates subjected to heat loads from the surrounding has been solved mathematically by Vodicka's method, which is a type of integral transform method, and completely explicit analytical solutions of the fluid temperature and local Nusselt number have been derived. The conclusions obtained through numerical calculations are summarised as follows:

(1) With regard to the Graetz problem in the case of a conduit between parallel plates, the number of partitions in the conduit should be over 20 in order to obtain a sufficiently accurate local Nusselt number.

(2) The local Nusselt number in the thermal entry region tends to increase with a decrease in the Biot number and power law model index.

(3) The effect of the power law model index i.e., velocity distribution of the fluid, on the critical fluid velocity for avoiding solidification is considerably smaller than that of the Biot number or Brinkman number.

\section{References}

[1] Vodicka, V., Linear heat conduction in laminated bodies. Mathematische Nachrichten, 14(1), pp. 47-55, 1955. (in German)

[2] Mikhailov, M.D. \& Ozisik, M.N., Unified analysis and solutions of heat and mass diffusion, Dover Publications: New York, pp. 344, 1994.

[3] Rosales, M.A. \& Frederick, R.L., Semi analytic solution to the Cartesian Graetz problem: results for the entrance region. International Communications in Heat and Mass Transfer, 31(5), pp. 733-740, 2004.

[4] Sadeghipour, M.S., Ozisik, M.N. \& Mulligan, J.C., Transient freezing of a liquid in a convectively cooled tube. Trans. ASME Journal of Heat Transfer, 104(2), pp. 316-322, 1982. 